\title{
Daring the Challenge and Thinking Big: The Value of Early Process R\&D
}

\author{
Stefan Abele ${ }^{a *}$, Jacques-Alexis Funela, Gunther Schmidta, Christian Moessner ${ }^{\mathrm{b}}$, \\ Mischa Schwaninger ${ }^{c}$, and Roger Marti ${ }^{d}$
}

Sandmeyer Prize 2015

\begin{abstract}
The production of the $\mathrm{L} / \mathrm{T}$ channel blocker ACT-280778 required the enantiomerically pure 5-phenylbicyclo[2.2.2]oct-5-en-2-one (1) as key building block. As the published routes towards $\mathbf{1}$ are very low yielding $(<0.5 \%$ yield) and comprise many steps that are not acceptable for scale-up, a series of processes to 1 was developed to match the increasing requirements from first kg-batches to clinical supplies. The three routes are characterized by an individual asset. (1) The first route contains a scale-up of a Diels-Alder reaction with highly reactive reagents and afforded $90 \mathrm{~kg}$ enantiomerically pure 1 . To mitigate safety risks, a flow reactor was developed for the high-temperature Diels-Alder reaction. This route relied on an efficient enantiomer separation on a $1 / 4$-ton scale by HPLC. (2) A Crystallization Induced Diastereomer Transformation (CIDT) during an intramolecular aldol reaction was the pivotal step of a first enantioselective route that starts with the Shibasaki reaction. (3) The $2^{\text {nd }}$ enantioselective route represents a rare example of organocatalysis on scale and allowed to skip six out of nine steps with a significant impact on the cost of goods. This simple way to 1 opened up a short synthesis of Hayashi's chiral diene ligands (bod $\left.{ }^{\star}\right)$ that were so far lacking an affordable access. Some of these novel C1-symmetrical dienes have shown very high enantioselectivities in Rh-catalyzed additions of arylboronates.
\end{abstract}

Keywords: Chiral dienes · Diels-Alder · Flow chemistry · Organocatalysis · Process safety $\cdot$ QbD $\cdot$ Scale-up

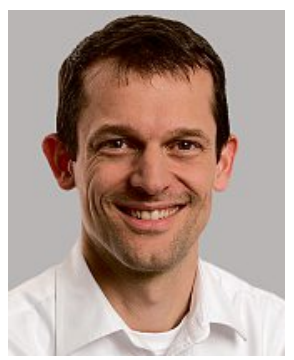

Stefan Abele studied chemistry in Germany, France, and Switzerland. He did his diploma work with Prof. Dr. R. R. Schmidt in Konstanz and obtained his PhD

${ }^{\star}$ Correspondence: Dr. S. Abele ${ }^{a}$ E-mail: stefan.abele@actelion.com ${ }^{a}$ Chemistry Process R\&D

Actelion Pharmaceuticals Ltd

Gewerbestrasse 16, $\mathrm{CH}-4123$ Allschwil

${ }^{b}$ Chemical Development and Catalysis

Solvias AG

Römerpark 2, $\mathrm{CH}-4303$ Kaiseraugst

Present address: Pharmaceuticals Division Chemical

Development \& Supply

F. Hoffmann-La Roche AG

'Swissi Process Safety $\mathrm{GmbH}$

Schwarzwaldallee 215, CH-4002 Basel

dHES-SO Haute école spécialisée de Suisse occidentale

Haute école d'ingénierie et d'architecture de Fribourg Institut ChemTech, Bd Pérolles 80, CH-1700 Fribourg under the guidance of Prof. Dr. D. Seebach at the ETH Zurich in 1999. For 7 years, he worked at Carbogen-Amcis, where he had the pleasure to perform or supervise the production of more than 80 Active Pharmaceutical Ingredients (API), in the roles of Project chemist, Group Leader $R \& D$, and Head GMP production. In addition, he was deputy Head ESH releasing production protocols at the Neuland site. In 2006, Stefan Abele set up a fully integrated Process $R \& D$ group at Actelion Pharmaceuticals Ltd with responsibility for all scientific, technical, organizational, and budgetary aspects. His teams'tasks encompass route finding and production of multi-kilogram amounts of API's, and the development of $2^{\text {nd }}$ generation routes, thereby combining scientific understanding and flexibility with commercial perspectives. Outsourcing parts of the work is driven by tactical or technological considerations and is deemed crucial for a lean and efficient drug development. He has authored around 60 scientific papers and patent applications, and he frequently gives invited lectures in industry, international conferences, and at universities.

„Es ist nicht genug zu wissen - man muss auch anwenden. Es ist nicht genug zu wollen-man muss auch tun. "__ Johann Wolfgang von Goethe

\section{Introduction}

The chiral bicycle ACT-280778 is a L/T calcium channel blocker potentially indicated for the treatment of hypertension and angina pectoris, currently under clinical development (Scheme 1). ${ }^{[1]}$ There was a need for a robust, safe, and scalable production of the Active Pharmaceutical Ingredient (API) ACT-280778 bis-maleate to supply material for preclinical and clinical studies.

Various technologies were required for the manufacturing of chiral bicycle $\mathbf{1}$ depending on the stage of development. Early stages call for a fit-for-purpose route that can safely deliver the API for first Entry-into-Man studies. A safe scale-up of a Diels-Alder reaction involving acrylonitrile monomers followed by a highly productive racemate resolution by largescale HPLC secured more than $90 \mathrm{~kg}$ of 1. Anticipating future demands, $2^{\text {nd }}$ generation routes were developed that obviated the intrinsic 50\%-yield loss of this racemate resolution by building on enantioselective steps. A final organocatalytic tandem Michael-aldol reaction of phenylacetaldehyde with cyclohexenone in the presence of proline brought down the cost of goods for 1 by more than $90 \%$. Besides, this simple approach to $\mathbf{1}$ opened up a short access to chiral dienes, a new class of privi- 
leged ligands that suffered from very high costs. Scheme 2 summarizes the salient features of our work en route to 1 that was honored by the Sandmeyer Prize 2015

FDA's recent imperative for Quality by Design (QbD) is not only decisive for patient safety but also for the timely and uninterrupted supply of drugs. In general, QbD means (i) the thorough understanding of the process and of the product and (ii) the knowledge of the risks associated with the manufacturing process and of the strategies how to mitigate those risks. More specifically, key ingredients of a $\mathrm{QbD}$ process are the assessment of the variability in the critical quality attributes, impurity tracking, detecting and solving mass-transfer related scale-up issues like mixing, etc. The ultimate goal is a robust process that delivers the API in a predictable and efficient way, in consistent purity and at acceptable costs. The route selection plays a primordial role: a route that calls for a plethora of control points and is strongly dependent on a narrow set of variables cannot be developed - through QbD principles - to the robustness level of a route that has intrinsic robustness. In other words, QbD should start as early as possible. An example of a less robust route is one with many telescoped steps lacking purification opportunity by crystallization, or a route that suffers scale limitations to control hazards (like the Diels-Alder batch reactions to 1). An attribute of an intrinsically robust route is for example little sensitivity to air, mixing, the quality of raw materials, or the simplicity of the process (like the organocatalytic approach to $\mathbf{1}$ ). It is an asset if a robust route is conceived and developed early. Expenditures and timeline until the registration of the process are likely to diminish, capitalizing on both the intrinsic robustness of the route and a thorough process understanding later on.

\section{The Diels-Alder Approach}

At the outset, Diels-Alder approaches seemed to be best suited for the quickest delivery of material for Phase I studies. Indeed, Paquette, [2] Takeuchi, ${ }^{[3]}$ and Hayashi[4] had published various accesses to 1 (Scheme 3).

However, already the first step, the Diels-Alder reaction of acetoxyacrylonitrile (4) and (cyclohexa-1,5-dien-1-yloxy)trimethylsilane $(\mathbf{5})$ en route to diketone rac-6 turned out to be inapplicable for the delivery of more than $50 \mathrm{~g}$ due to tar formation, low purity and yield, all being aggravated by non-solid, non-UV active intermediates. Furthermore, the very low yield was an impediment for scale-up. We homed in on testing many different approaches towards 1, Scheme 4 summariz-

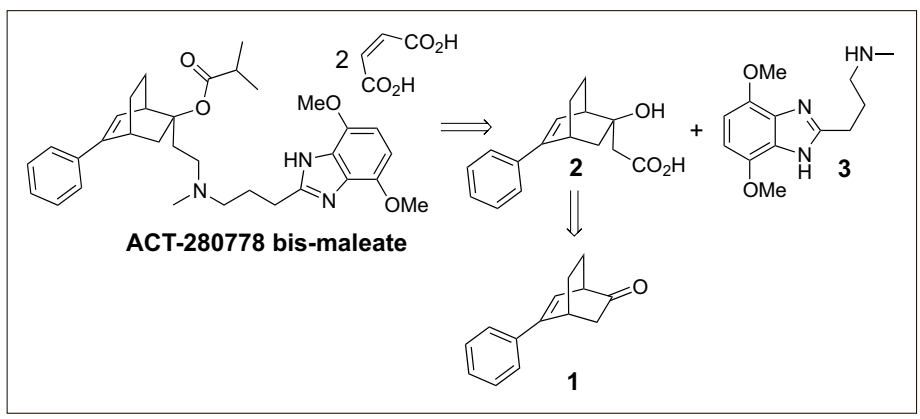

Scheme 1. Retrosynthesis of ACT280778 to afford bicyclic ketone $\mathbf{1}$, its enolate addition product 2 , and benzimidazole $\mathbf{3}$ as key building blocks.

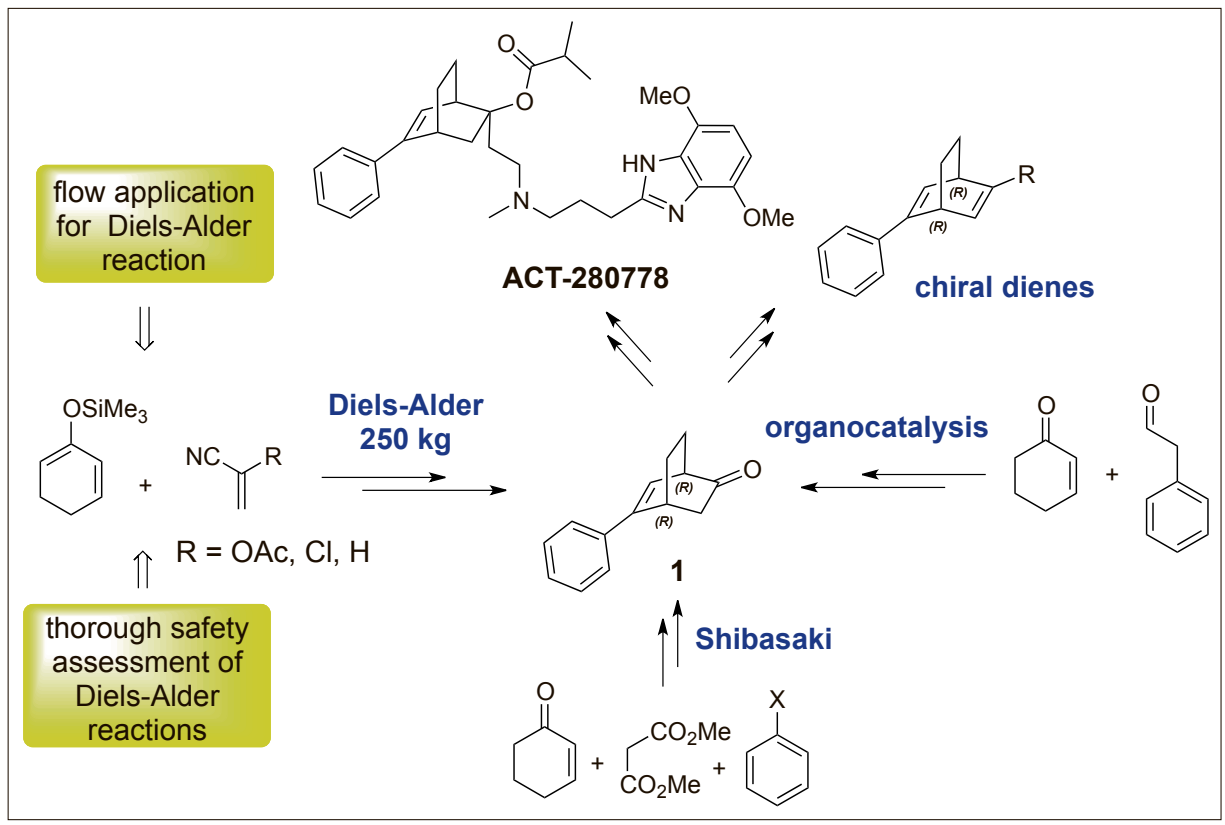

Scheme 2. Synopsis of the key technologies used to access the chiral bicyclic ketone 1 , as building block for the manufacture of the L/T channel blocker ACT-280778.

es those that did deliver the target bicyclic ketone. Routes a)-d) rely on Diels-Alder reactions, whereas routes e)-i) build upon asymmetric Michael additions. The three Diels-Alder reactions of the TMS-diene $\mathbf{5}$ with the acrylic monomers $\mathbf{4 , 6}$, and 7 were developed as fit-for-purpose processes to deliver from $2-180 \mathrm{~kg}$ racemic 1 that was later on resolved into its enantiomers by HPLC (vide infra). ${ }^{[5]}$ An intriguingly short access to 1 relied on the Diels-Alder reaction of 3,4-dihydro-1,1'-biphenyl (8) with 4. However, 8 was not stable and its onset temperature for the highly exothermic decomposition in the Differential Scanning Calorimetry (DSC) coincided with the process temperature of $110^{\circ} \mathrm{C}$. The Michael addition of either malonates or phenylacetonitrile to cyclohexenones $\mathbf{9}, \mathbf{1 0}$, or 11 was a complementary method to build

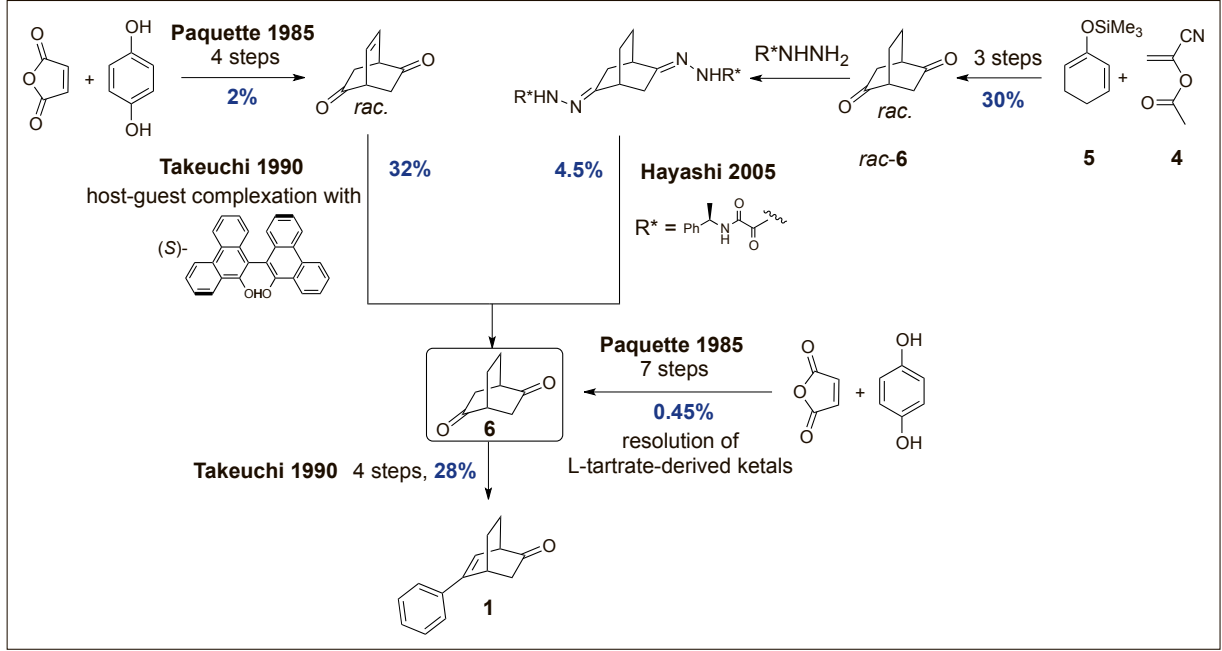

Scheme 3. Published routes to $\mathbf{1}$, all converging in bicyclic diketone 6 . 


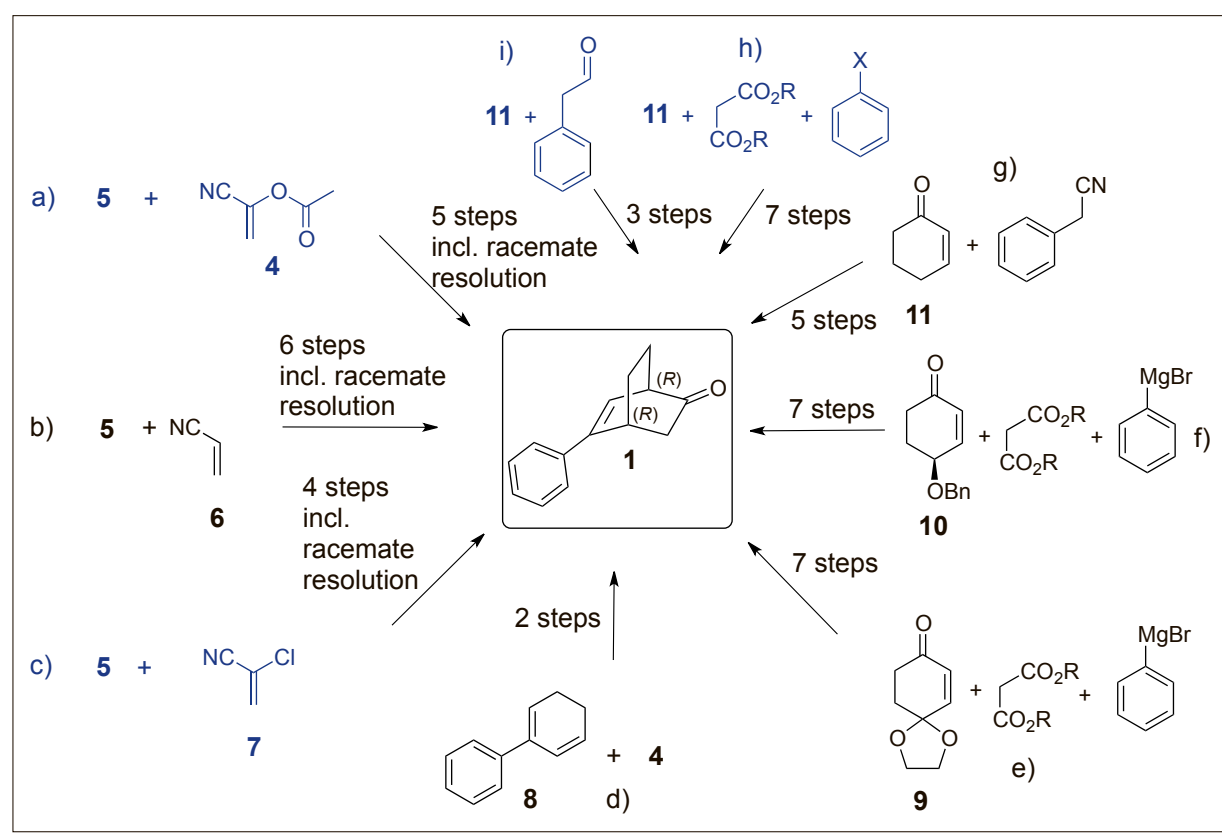

Scheme 4. Various approaches tested that were affording 1. Methods a), c), h), and i) have been demonstrated on 100-g to 180-kg scale (highlighted in blue).

up the bicycle. Especially approach h) was of importance as the Shibasaki reaction, ${ }^{[6]}$ i.e. the asymmetric Michael addition of dimethylmalonate to cyclohexenone, established the first chiral center in an efficient manner and allowed for the first enantioselective approach to $1,{ }^{[7]}$ and ultimately paved the way to the final organocatalytic approach i), ${ }^{[8]}$ both described as $2^{\text {nd }}$ generation routes below.

Further approaches were tested without success. Diels-Alder reactions of various alkynes with $\mathbf{5}$ gave no reaction or led to the Alder-Rickert product. Lewis acids are typically used to accelerate DielsAlder reactions. However, the TMS-diene 5 proved to be too labile for many of them, and none of the tested Lewis acids allowed a reduction of the high reaction temperatures $\left(100-140{ }^{\circ} \mathrm{C}\right)$. Asymmetric Diels-Alder reactions were screened with different families of chiral catalysts, dienophiles and dienes, but led to a complicated mixture of diastereoisomers with low ee's of the desired isomers.

For first urgent material deliveries, the neat Diels-Alder reaction of acetoxyacrylonitrile (4) with diene 5 at $140{ }^{\circ} \mathrm{C}$ could be exploited thanks to a new twist in the reaction sequence (Scheme 5). Original reports hydrolyzed the Diels-Alder product $\mathbf{1 2}$ to the symmetrical diketone rac-6 where the two ketone functions are not differentiated. We noticed that $\mathbf{1 2}$ could be selectively ketalized to $\mathbf{1 3}$ thus paving the way to monoprotected ketone 14. Addition-elimination with $\mathrm{PhMgBr}$ afforded $\mathrm{rac}-\mathbf{1}$ that was separated on chiral stationary phase with a surprisingly high productivity. As the delivery time of both the diene $\mathbf{5}$ and the dienophile 4 was several months (and the costs being $\$ 8^{\prime} 000 / \mathrm{kg}$ ), both were pro- duced in-house to enable the crucial start. Acetoxyacrylonitrile was produced from chloroacetaldehyde (15) and $\mathrm{NaCN}$ via 2-chloro-1-cyanoethyl acetate (16).

Severe safety concerns about both the dienophiles (decomposition energies in the range of $1 / 3$ of that of trinitrotoluene) and the reaction hampered an immediate and safe scale-up. The rapid delivery of this first $\mathrm{kg}$ of $\mathbf{1}$ was dependent on an early risk assessment of the hazardous Diels-Alder reactions with highly reactive acrylic monomer $\mathbf{4}$ by the Swissi Process Safety GmbH. ${ }^{[9,10]}$ Fig. 1 shows the DSC trace of the neat Diels-Alder reaction of 4 and 5. The first exothermic event with a peak around $180{ }^{\circ} \mathrm{C}$ can be attributed to the desired reaction, and overlaps with the exothermic decomposition of the product 12. Further safety investigations restricted the scale of this reaction at the low $\mathrm{kg}$ range in agitated glass vessels. ${ }^{[9]}$

We hence replaced acetoxyacrylonitrile (4) by other dienophiles like acrylonitrile (6) or chloroacrylonitrile (7). The DielsAlder reaction of $\mathbf{6}$ with 5 suffered from the same limitations as the one with 4 . Fortunately, the DSC trace of a mixture of 7 with the diene $\mathbf{5}$ showed that these two exothermic events are better separated (Fig. 2). However, when the neat Diels-Alder reaction with 7 was performed at $70{ }^{\circ} \mathrm{C}$ instead of $140{ }^{\circ} \mathrm{C}$ ( 7 being more reactive than $\mathbf{5}$ ), the adiabatic end temperature would be $270{ }^{\circ} \mathrm{C}$, still triggering decomposition.

Further thermal risk assessment was mandatory using the concept of the cool-

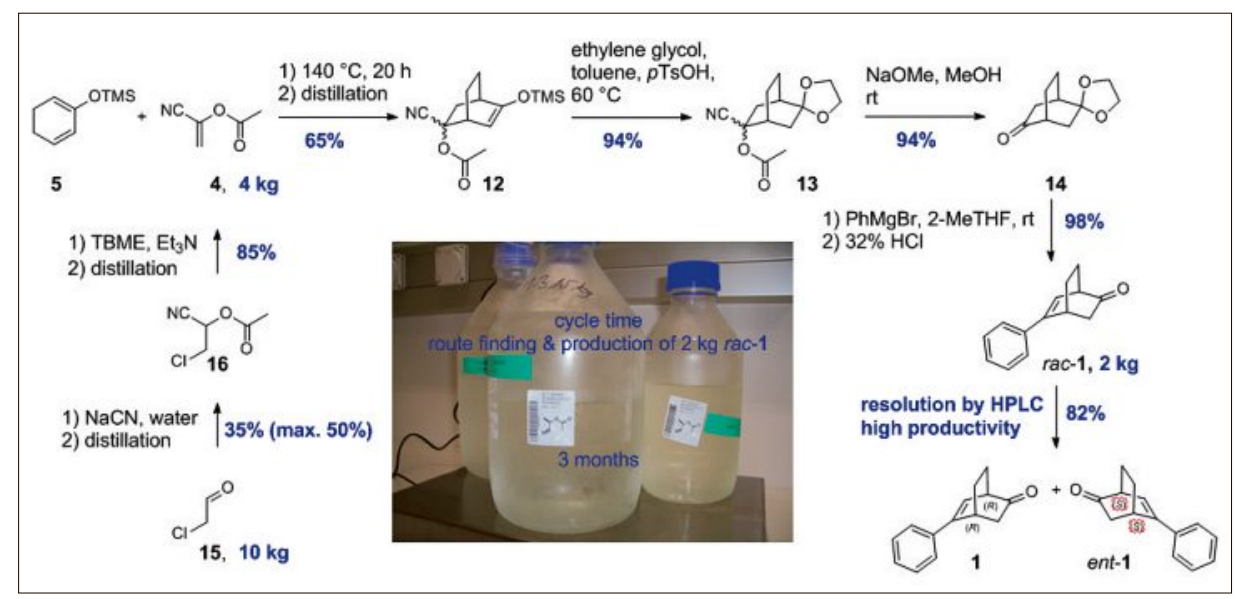

Scheme 5. Acetoxyacrylonitrile approach to secure early tox batch supplies.

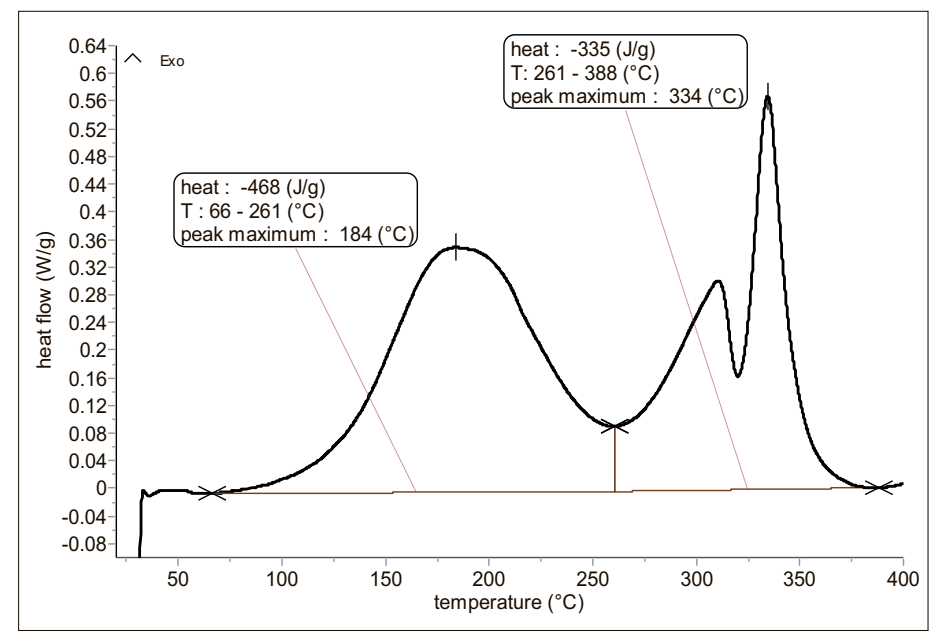

Fig. 1. DSC trace of a mixture of $\mathbf{4}$ and $\mathbf{5}$ $(1: 1)$ at $4 \mathrm{~K} / \mathrm{min}$. Heat of desired reaction $Q_{r}$ $=-468 \mathrm{~kJ} / \mathrm{kg}(68-261$ $\left.{ }^{\circ} \mathrm{C}\right)$; heat of decomposition $Q_{d}=-335$ $\mathrm{kJ} / \mathrm{kg}\left(261-388^{\circ} \mathrm{C}\right)$. 


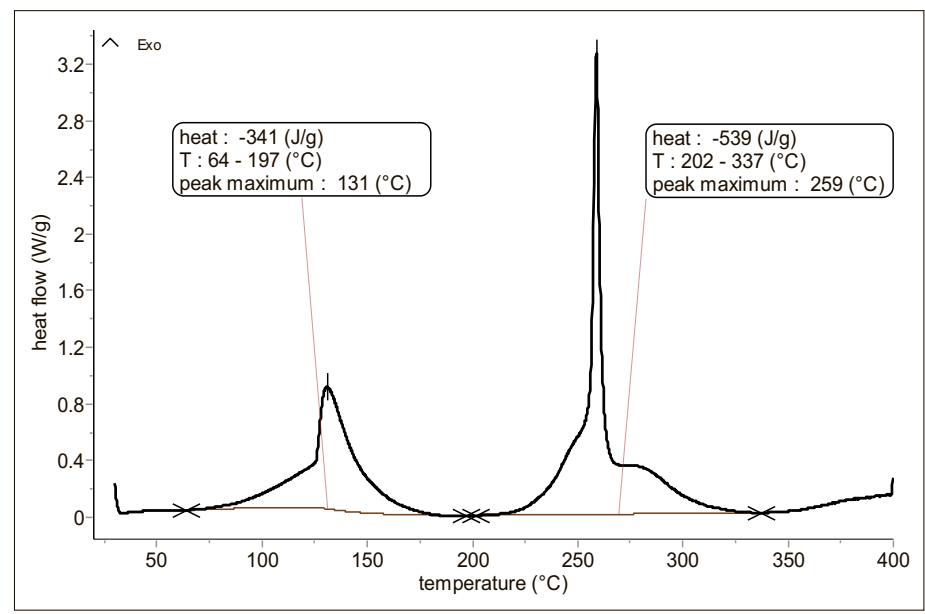

Fig. 2. Screening DSC trace of a mixture of 7 and 5 ( 1.12 equiv. $\mathbf{5}$, with 0.3 equiv. $\mathrm{NaHCO}_{3}, 0.01$ equiv. TEMPO) at $4 \mathrm{~K} / \mathrm{min}$, recorded right after preparation of the mixture. $Q_{\mathrm{r}}=-341 \mathrm{~kJ} /$ $\mathrm{kg}\left(64-197^{\circ} \mathrm{C}\right) ; \mathrm{Q}_{\mathrm{d}}=$ $-539 \mathrm{~kJ} / \mathrm{kg}(202-337$ $\left.{ }^{\circ} \mathrm{C}\right)$.

ing failure scenario. ${ }^{[10]}$ We required information about the Time to Maximum Rate under adiabatic conditions (TMR ${ }$ ), derived from isothermal DSC measurements, and information about the Maximum Temperature of the Synthesis Reaction (MTSR). Fig. 3 shows the batch reaction of $\mathbf{4}$ and $5(213 \mathrm{~g})$ in a reaction calorimeter and indicates that the temperature which can be reached after a cooling failure $\left(T_{\mathrm{cf}}\right)$ passes its maximum at MTSR $=245{ }^{\circ} \mathrm{C}$ after $2.2 \mathrm{~h}$. At this time, the batch temperature is $114^{\circ} \mathrm{C}$ with a thermal conversion of $15 \%$. The Diels-Alder reaction of $\mathbf{4}$ with 5 is run in full-batch mode without solvent barrier. At MTSR $=245{ }^{\circ} \mathrm{C}$, decomposition would set in immediately (see DSC), leading to a final temperature of $>400{ }^{\circ} \mathrm{C}$.

The reaction calorimetric data of $\mathbf{5}$ and 7 led to a similar picture, i.e. decomposition would be triggered in case of a cooling failure. Therefore, the Diels-Alder reaction was diluted with toluene to mitigate the adiabatic temperature rise. Fig. 4 shows reaction calorimetry of the diluted batch process with $\mathbf{5}$ and $\mathbf{7}$ at $80{ }^{\circ} \mathrm{C}$. Now, the MTSR was lowered to $132^{\circ} \mathrm{C}$ as compared to $250{ }^{\circ} \mathrm{C}$ in the undiluted case, and the probability of a runaway was significantly reduced based on modeling of the kinetics. ${ }^{[9]}$ The diluted Diels-Alder reaction of $\mathbf{7}$ and $\mathbf{5}$ to the bicycle $\mathbf{1 8}$ was deemed safe for a scale of $100 \mathrm{~kg}$ reaction mass in the reactor. An adiabatic situation in the worst case, i.e. at the end of the heat ramp, would result in a MTSR of $132{ }^{\circ} \mathrm{C}$. The boiling of toluene starts already at $112{ }^{\circ} \mathrm{C}$, acting as a thermal barrier. This Diels-Alder reaction was reproduced several times on 54-147 $\mathrm{kg}$ sized batches in a 200-L Hastelloy reactor giving similar yields and purities.

During the early phase of route scouting, the Diels-Alder reaction of acrylonitrile with 5 was one of the first approaches with a chance for scale-up. As the maximal scale of the Diels-Alder reaction was still limited to 100-kg batch size based on hazard assessment, we developed a flow application as a contingency (Fig. 5). The target was to keep technical complexity low in order to be able to run this at many locations in a robust manner. The final neat continuous Diels-Alder reaction of the diene 5 with acrylonitrile $\mathbf{6}$ was performed

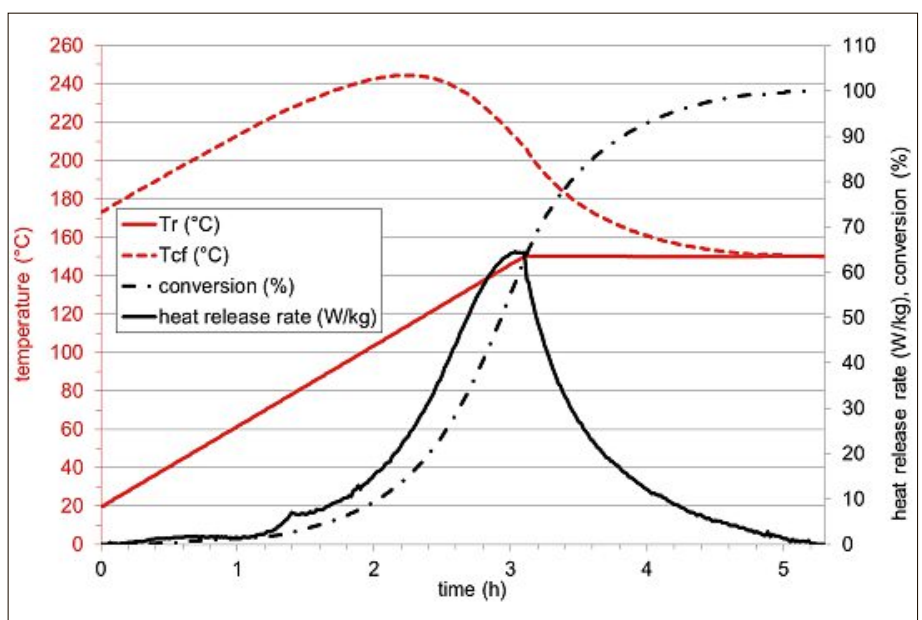

Fig. 3. Reaction calorimetric data of the neat batch process of $\mathbf{4}$ and $\mathbf{5}$. Heat rate: $0.7 \mathrm{~K} / \mathrm{min}$ from $20-150{ }^{\circ} \mathrm{C}$, then $2 \mathrm{~h}$ isothermal at $150^{\circ} \mathrm{C}$. The figure shows the reactor temperature $T_{r}$ $\left({ }^{\circ} \mathrm{C}\right), \mathrm{T}_{\text {cf }}\left({ }^{\circ} \mathrm{C}\right)$, the thermal conversion (\%), and the heat release rate $(\mathrm{W} / \mathrm{kg})$ as a function of time. Heat of reaction $Q_{r}=-308 \mathrm{~kJ} /$ $\mathrm{kg}, \mathrm{MTSR}=245^{\circ} \mathrm{C}$.

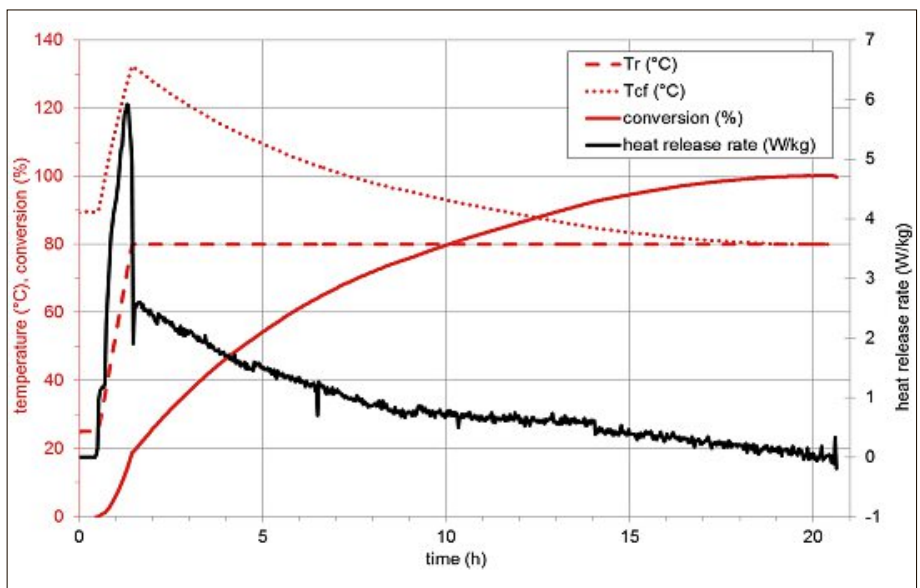

Fig. 4. Reaction calorimetry of the diluted batch process with 5 and 7 at $80^{\circ} \mathrm{C}$ (34\% $\mathrm{w} / \mathrm{w}$ in toluene). Reaction heat $=-111$ $\mathrm{kJ} / \mathrm{kg}, \mathrm{MTSR}=132$ ${ }^{\circ} \mathrm{C}$.

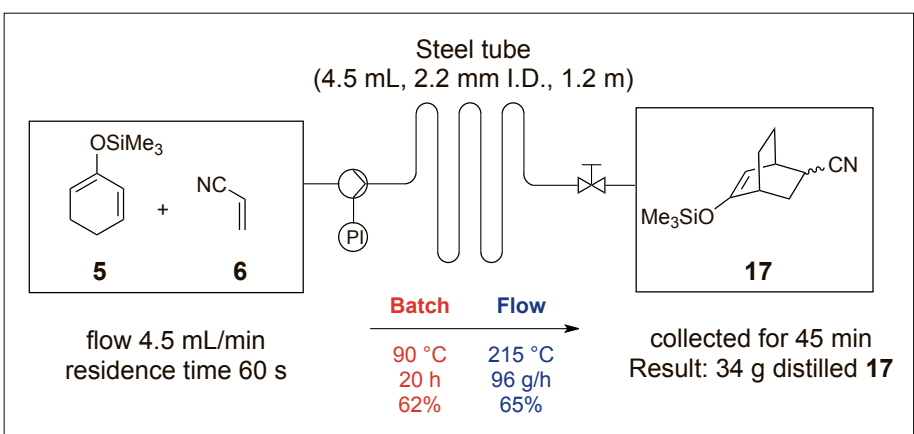

Fig. 5. Flow reactor. 


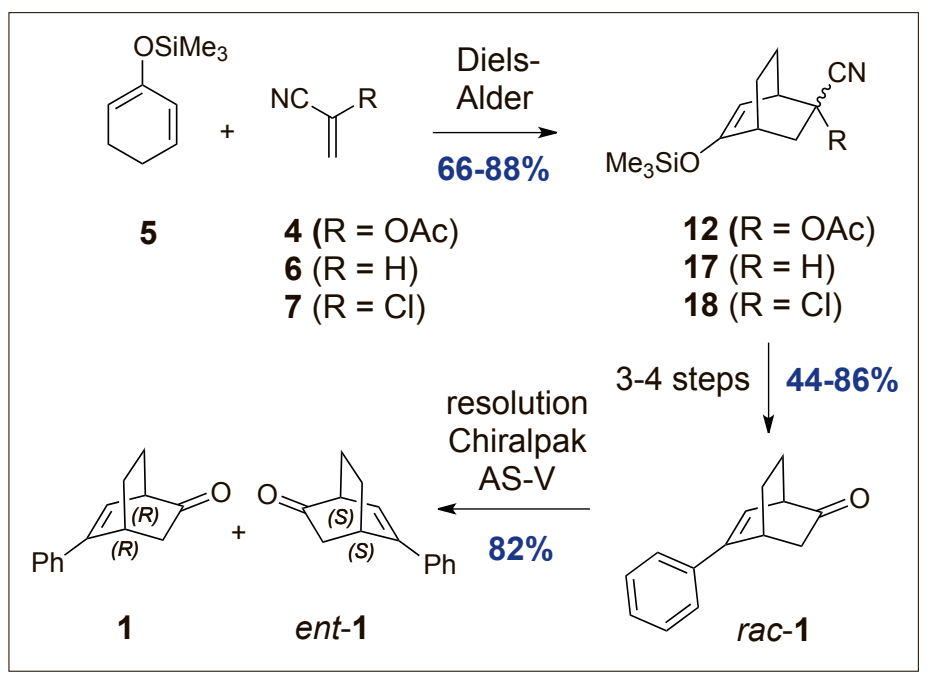

Scheme 6. DielsAlder approaches to phenylketone 1 with a resolution of rac-1 on 180-kg scale.

our own experience was far from extensive in this field. Based on the rare use of the Diels-Alder reaction at industrial scale, process chemists seem to lock up the Diels-Alder reaction a priori into the field of 'non-scalable' transformations. We endeavoured to scout for large-scale applications of this highly atom-efficient reaction and were pleased to find more than 160 examples from pharma, agrochemical, and fragrance industry. Our work illustrates the benefit of overcoming such prejudice. ${ }^{[13]}$

\section{Assembling the Drug Substance ACT-280778}

With the required chiral building block 1 at hand, the downstream chemistry to the API ACT-280778 could be started. The route development and $\mathrm{kg}$-production of the benzimidazole side chain $\mathbf{3}$ and the last steps to the API have been described in detail.[14] In the following, we summarize the salient features of the final process.

\section{Benzimidazole 3}

Benzimidazole $\mathbf{3}$ was derived from cheap starting materials: 1,4-dimethoxybenzene (19), nitric acid, and N-methylpyrrolidinone (NMP, Scheme 7). It is noteworthy that all atoms of the solvent NMP end up in the building block, rendering this approach highly atom-efficient as compared to the classical two-step buildup of the benzimidazole. The nitration of 19 occurred in moderate regioselectivity to give the highly energetic dinitro compound $\mathbf{2 0}^{[15]}$ in 100-kg batches (it was not shock sensitive according to falling hammer test). The undesired 1,4-dinitro isomer of $\mathbf{2 0}$ was successfully purged after hydrogenation over $\mathrm{Pd} / \mathrm{C}$ to the phenylene diamine $\mathbf{2 1}$, most probably via formation of para-chinoid intermediates forming side products that ultimately remained on the filter cake. The filtrate containing $\mathbf{2 1}$ was highly sensitive to air: it rapidly formed a black tarry product. By way of $\mathrm{HCl}$ salt formation, the purity (>99\% a/a and w/w) and stability were highly improved. The benzimidazole 3 was formed by heating $\mathbf{2 1} \cdot \mathrm{HCl}$ with $20 \% \mathrm{HCl}$ in NMP at $102-104{ }^{\circ} \mathrm{C}$ for a day. Like the phenylene diamine $\mathbf{2 1 , 3}$ is highly water soluble and required the polar, not water-immiscible solvent $n$-butanol for extraction. Residual tarry by-products were removed by a charcoal treatment. The best crystalline form of $\mathbf{3}$ that was both stable and allowed for the highest upgrade in purity was the mono-hydrate of the dihydrochloride salt. Its reproducible production required a careful azeotropic adjustment of the water content (2.5-3.5\%) during the reactive crystallization from butanolwater. A total of $141 \mathrm{~kg}$ of $32 \mathrm{HCl}$ was successfully produced as off-white powder ( $99.5 \%$ a/a (HPLC), $99.2 \%$ w/w (NMR assay), ash $<1 \%$ w/w, Pd $<0.1 \mathrm{ppm})$.

\section{Last Steps Towards ACT-280778}

En route to the API ACT-280778, the key challenges were the creation of the tertiary alcohol $\mathbf{2}$, the oily nature of most intermediates, and the identification of a suitable solid form of the API (Scheme 8). The addition of the enolate of tert-butylacetate to $\mathbf{1}$ at $-78{ }^{\circ} \mathrm{C}$ gave $\beta$-hydroxyester 22 in a diastereomeric ratio (dr) of 2.2 to 3.3:1. This moderate endo-selectivity can be attributed to low difference in steric bulk of the $\mathrm{CH}_{2}-\mathrm{CH}_{2}$ bridge and the $\mathrm{CH}=\mathrm{CH}$ bridge. We took profit of the crystallinity of $\mathbf{2}$ that allowed for an efficient upgrade of the purity, thus purging the undesired diastereoisomer. Towards this end, the toluene solution of intermediate hydroxy ester 22 was solvent exchanged to ethanol, the solvent for the ensuing ester hydrolysis to 2 . Extraction of the quite water-soluble $\beta$-hydroxy acid $\mathbf{2}$ was best done with 2-MeTHF and a controlled solvent swap to EtOAc led to crystallization of $\mathbf{2}$ with excellent dr, keeping the enantiomeric ratio (er) of ketone $\mathbf{1}$ unchanged. Amide bond formation with the benzimidazole dihydrochloride $\mathbf{3} 2 \mathrm{HCl}$ to $\mathbf{2 3}$, followed by reduction of the tertiary amide with $\mathrm{LiAlH}_{4}$ gave amine $\mathbf{2 4}$ as foam. This was subjected to isobutyroyl chloride and led to over-acylation with the acylation at the undesired nitrogen of the benzimidazole being kinetically favored. The overacylated intermediate was treated with $\mathrm{NaOMe}$ to afford crude ACT-280778. As the free form of the API is also a foam, the first syntheses of the API were plagued by the absence of opportunities for scaleable purifications like crystallization. To this end, we identified the bis-oxalate salt of $\beta$-hydroxy amine $\mathbf{2 4}$ as a suitable solid that allowed byproducts to be removed from the amide formation and the reduction step. In-depth screening of pharmaceutically acceptable salts of the API finally culminated in the bis-maleate of ACT-280778 that fulfilled the requirements like purity upgrade, stability, good

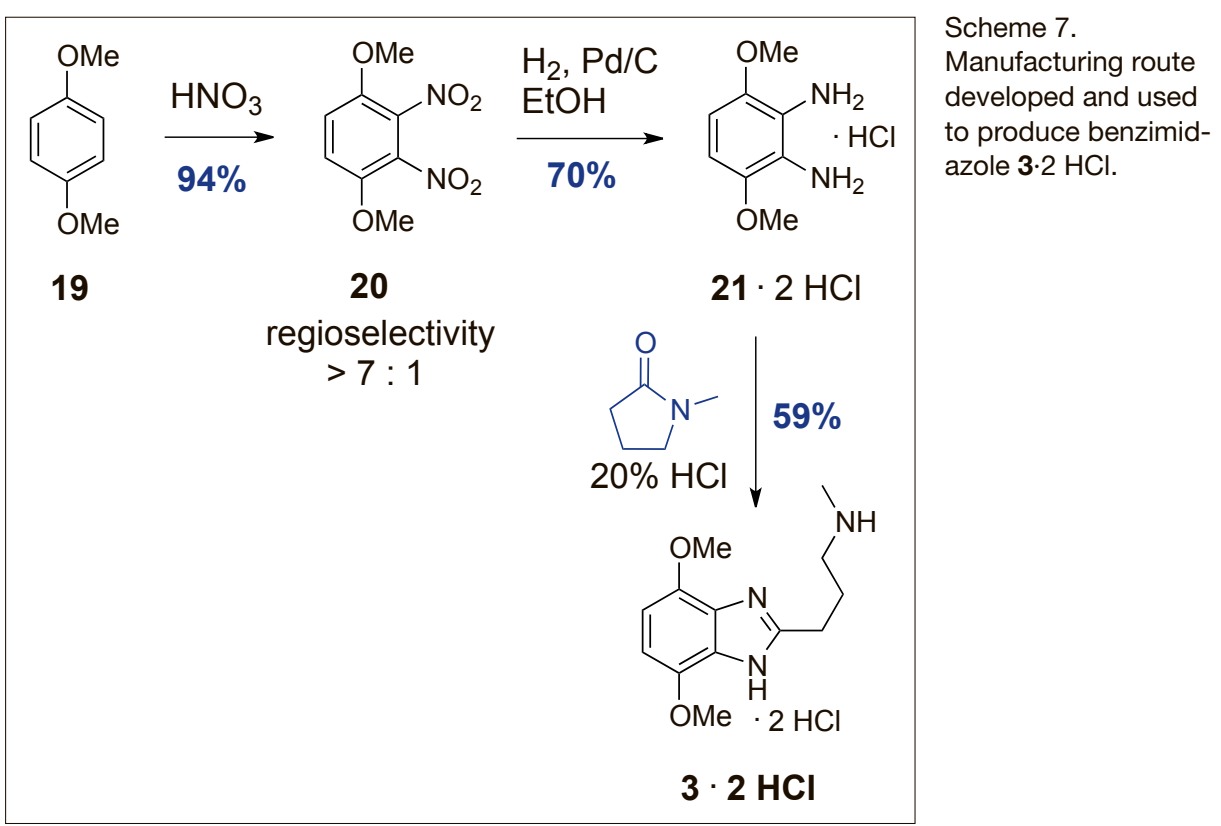




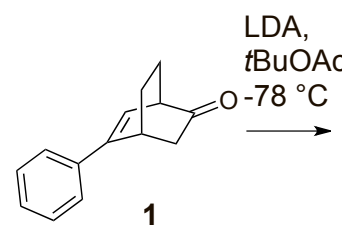

er $98: 2$

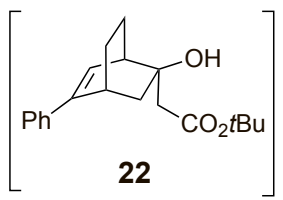

dr 2.2 to $3.3: 1$
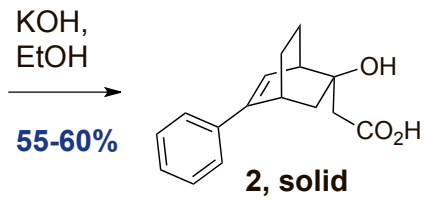

$d r>99: 1$, er $>98: 2$

EDCl, $\mathrm{HOBt}$ $3 \cdot 2 \mathrm{HCl}$<smiles>CCOc1ccc(OC)c2[nH]c(CCCN(C)CCC3(O)C4CC(c5ccccc5)CC3C4)nc12</smiles>

24 bis-oxalate

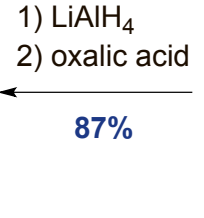

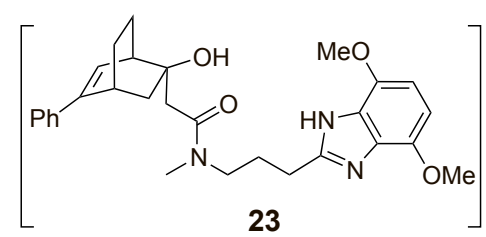

23
1) $\mathrm{iBuCOCl}$

2) $\mathrm{NaOMe}$

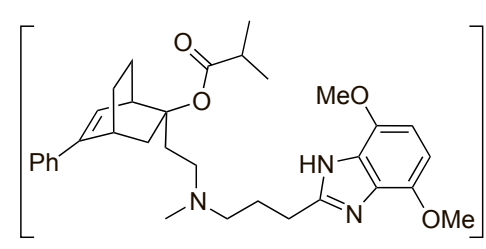

ACT-280778 maleic acid

$73 \%$

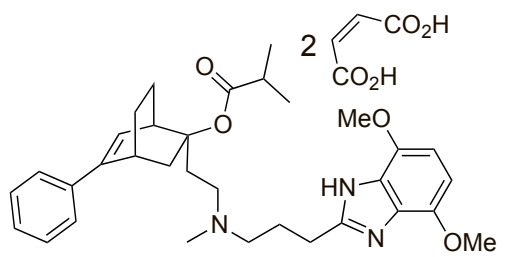

ACT-280778 bis-maleate
Scheme 8. Last seven steps toward $14 \mathrm{~kg}$ of API ACT-280778 bis-maleate with indicated telescoped steps.

filterability, easiness of drying, and compatibility with the formulation with excipients. Overall, this synthetic sequence delivered $14 \mathrm{~kg}$ of the API ACT-280778 for clinical tests with a yield of $38 \%$ (7 linear steps) from chiral bicyclic ketone $\mathbf{1}$, and $17 \%$ yield (14 linear steps) from cyclohexenone.

\section{$2^{\text {nd }}$ Generation Routes ${ }^{[16]}$}

The Diels-Alder approach (Scheme 6) delivered enough ketone $\mathbf{1}$ for the manufacture of time critical API for clinical Phase 1 supply. An enantioselective route was required for larger scales to remove the burden of the enantiomer separation on large scale without the possibility of recycling the undesired enantiomer. Two practical routes to 1 have been developed, both relying on catalytic enantioselective catalysis.

\section{$2^{\text {nd }}$ Generation Route 1, Relying on a CIDT Strategy (Shibasaki)}

The first approach to $\mathbf{1}$ makes use of the Shibasaki reaction to efficiently install one bridge head stereocenter in malonate 25, ${ }^{[6]}$ and features a Crystallization Induced Diastereomer Transformation $(\text { CIDT })^{[17]}$ as pivotal step delivering the crystalline intermediate $(1 R, 4 R, 5 S, 6 S)$-6-
Homobenzylic alcohol 26 was oxidized to ketal aldehyde 27 with the TEMPO-bleach hydroxybicyclo[2.2.2] octan-2-one 28a via of a $1-\mathrm{kg}$ batch of $\mathbf{1}$ in a 30-L reactor in less

The details of the last steps to $\mathbf{1}$ on $\mathrm{kg}$-scale are highlighted in Scheme 10. han 10 working days. protocol. To account for the phenylacetaldehyde's (27) limited stability, it was telescoped in EtOAc solution into the aldol cyclization step that ran in the presence of $32 \% \mathrm{HCl}$ at $50{ }^{\circ} \mathrm{C}$ for $2 \mathrm{~h}$. Cooling to $0{ }^{\circ} \mathrm{C}$, filtration and washing afforded bicyclic alcohol 28a as one single diastereoisomer in excellent er as a white crystalline solid. The dehydration to 1 using standard methods produced black tarry mixtures. ${ }^{[7]}$ The bicyclic system is suspected to be prone to skeletal rearrangements. After extensive scouting, a fine-tuned two-step process turned out to be optimal. Alcohol 28a was transformed into its mesylate $\mathbf{2 9}$ that was telescoped into the E2 syn elimination in 2,4,6-collidine at $143-150{ }^{\circ} \mathrm{C}$ for $1 \mathrm{~h}$. Neutralization, extraction into heptane and concentration led to crystallization of the ketone $\mathbf{1}$ in high purity. Crystallization of 1 proved challenging due to the low melting point $\left(66-68^{\circ} \mathrm{C}\right)$ and its lipophilic and unpolar nature. Control of the purity of $\mathbf{2 8 a}$ was crucial for the ensuing dehydration sequence to 1: phenylketone 1 of inferior quality (i.e. $<90 \%$ a/a or w/w) was impossible to crystallize without oil formation.

A striking improvement in color and habitus of 1 was achieved with this new route when compared with the batches derived from the Diels-Alder approach followed by racemate resolution by HPLC (Fig. 6).

A mechanistic understanding of the strategic aldol cyclization from ketalaldehyde 27 to the secondary bicyclic alcohol 28a was crucial for the success of this approach. Three isomers were isolated (28a, 28b, and 28c) and their structure proven by X-ray crystal structure analysis. Subjecting these isomers separately to the dehydration to 1 led to the following observations: (i) 28c did not react in the elimination sequence; (ii) $\mathbf{2 8 a}$ and $\mathbf{2 8 b}$ were comparable in reactivity (kinetics \& purity);

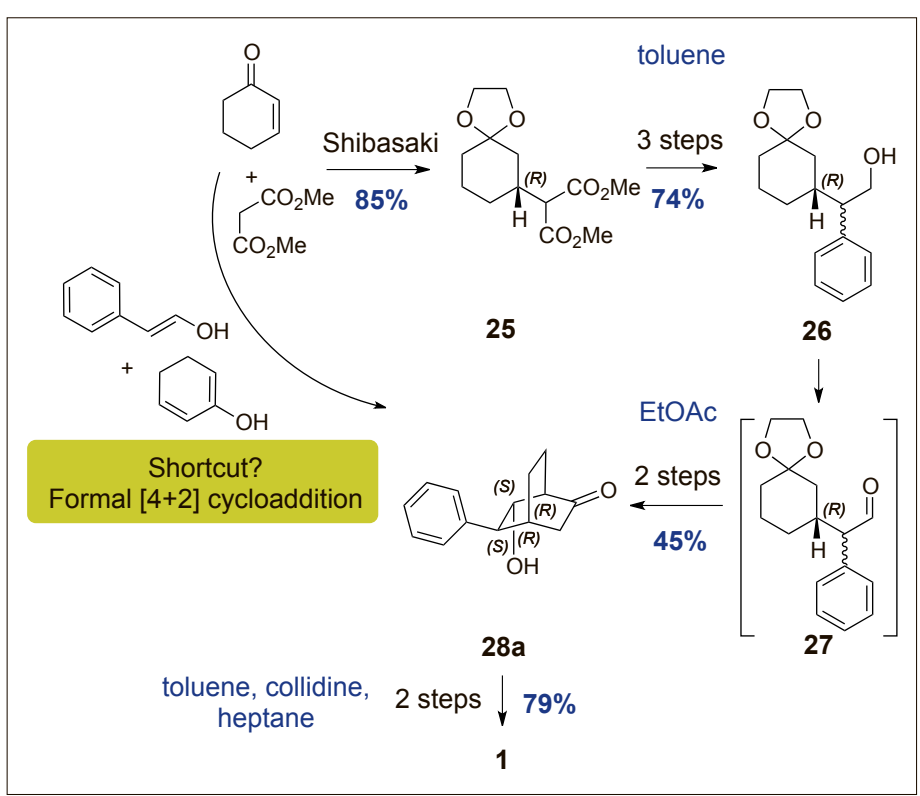

Scheme 9. Nine-step $2^{\text {nd }}$ generation route 1 (Shibasaki approach). The solvents used for the telescoped steps are highlighted in blue. Potential shortcut via a formal Diels-Alder reaction is shown in yellow $\left(2^{\text {nd }}\right.$ generation route 2 , vide infra). 


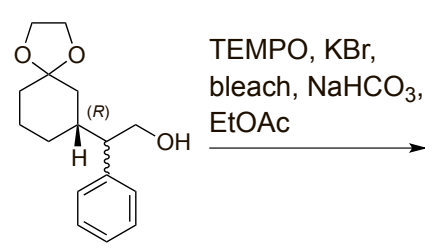

26

$\operatorname{dr} 60: 40$

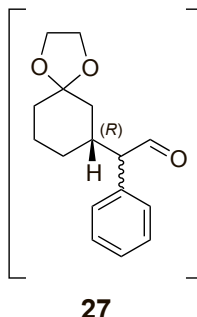

dr 60:40
1) 0.3 eq $32 \% \mathrm{HCl}$

$50{ }^{\circ} \mathrm{C}, 2 \mathrm{~h}$

2) $\mathrm{cool} 0^{\circ} \mathrm{C}$

3) filter

4) wash EtOAc

5) dry

$46 \%$ ( 2 steps)

3-kg scale

kept in EtOAc solution

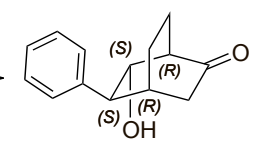

28a

$>99 \% \mathrm{w} / \mathrm{w}$ er $>99.5: 0.5$

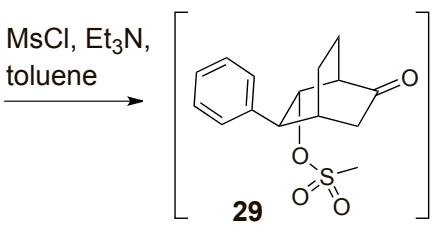

solvent swap from

toluene to 2,4,6-collidine

\section{1) 2,4,6-collidine, $143-150{ }^{\circ} \mathrm{C}, 1 \mathrm{~h}$ \\ 2) $1 \mathrm{~N} \mathrm{HCl}$, heptane 3) cryst. heptane}

$80 \%$ (2 steps)

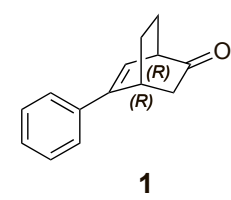

$100 \% \mathrm{w} / \mathrm{w}$ er $>$ 99.5:0.5
Scheme 10. The last four steps on $\mathbf{k g}$-scale from alcohol $\mathbf{2 6}$ towards enantiomerically pure $\mathbf{1}$ with one isolated intermediate.
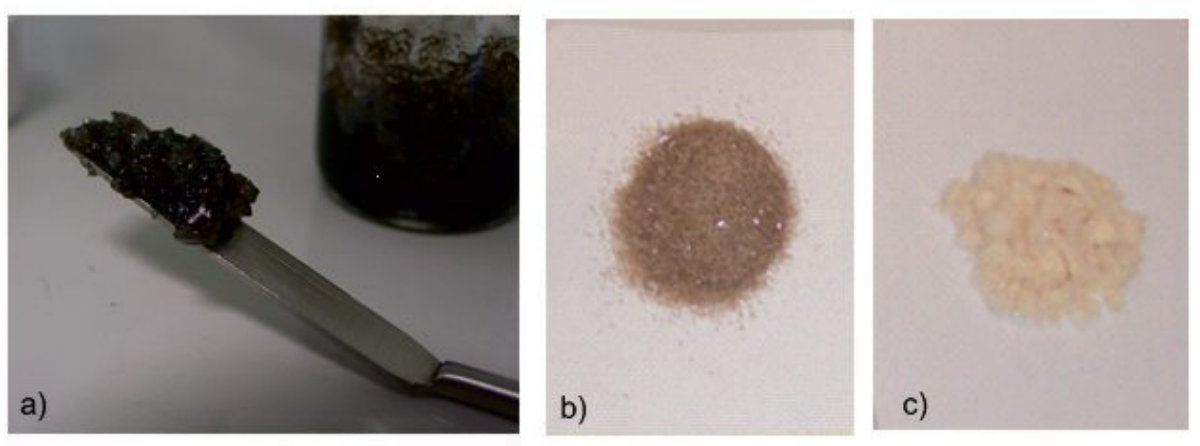

Fig. 6. Color and habitus of 1. (a) from Diels-Alder route after racemate resolution by HPLC on 180-kg scale; from 'Shibasaki' route, recrystallized from (b) heptane or (c) heptane/TBME. mer, a thermodynamic sink. 'Traditional' measurement of melting points ( $\mathrm{mp}$ ) of the main isomers was key to design this CIDT (typically a high mp leads to low solubility).

\section{$2^{\text {nd }}$ Generation Route 2, Organocatalysis}

Scheme 9 reveals a daring shortcut relying on a formal $[4+2]$ cycloaddition of phenylacetaldehyde and cyclohexenone, thus removing 6 out of the 9 steps. The same chiral intermediate $\mathbf{2 8 a}$ could conceivably be obtained in an organocatalytic tandem Michael addition-aldol reaction from 2-cyclohexenone and phenylacetaldehyde in the presence of an organocatalyst. A publication by Bella et al. ${ }^{[18]}$ inspired us to screen organocatalysts (4), solvents (5), and additives (13) under various reaction conditions with Solvias AG. We could build upon our expertise with the intramolecular aldol cyclization and CIDT gathered during the development of $2^{\text {nd }}$ generation route 1 , allowing us to isolate 28a in essentially pure form and good yield out of a reaction mixture containing a wealth of byproducts and isomers. L-Proline proved to be the best organocatalyst in terms of activity and selectivity: a maximum of er was reached at 72:28 when the reaction was run in the presence of Hünig's base in toluene at $45{ }^{\circ} \mathrm{C}$ for $4 \mathrm{~d}$, whereas the er was 86:14 when the reaction was run at $0{ }^{\circ} \mathrm{C}$ at the cost of a 21-day reaction. The dr (28a:28b) started at 50:50 and grew to $>92: 8$ at the end of the reaction, pointing to another nice example of a CIDT for this tandem Michael-aldol reaction with many products in equilibrium. The enantiomeric (iii) 28a was obtained in highly pure form by simple filtration of reaction. These results guided us to develop this transformation into a CIDT with 28a as target isomer for the screening of reaction parameters. On industrial scale, a CIDT represents a 'dream' reaction as the reaction, the isomerization (via the enol of the phenylacetaldehyde intermediate), and the crystallization occur in just one vessel without the need for additional downstream unit operations like extractive workup. Scheme 11 depicts the observed diastereoisomeric ratios of both starting material (27, the enol form of the deprotected ketone of $\mathbf{2 7}$ is drawn for clarity) and isomeric products 28 of the intramolecular aldol reaction. In-process controls of reaction mixtures indicate that a plethora of conceivable intermediates (not shown) were formed after 20-30 min. When the reaction was aged at $50{ }^{\circ} \mathrm{C}$ for $2 \mathrm{~h}$, two isomers, $\mathbf{2 8 a}$ and $\mathbf{2 8 b}$ prevailed. The success of this transformation relies on the precipitation of the least soluble 28a from the reaction mixture, thus pushing the equilibrium to this desired iso-

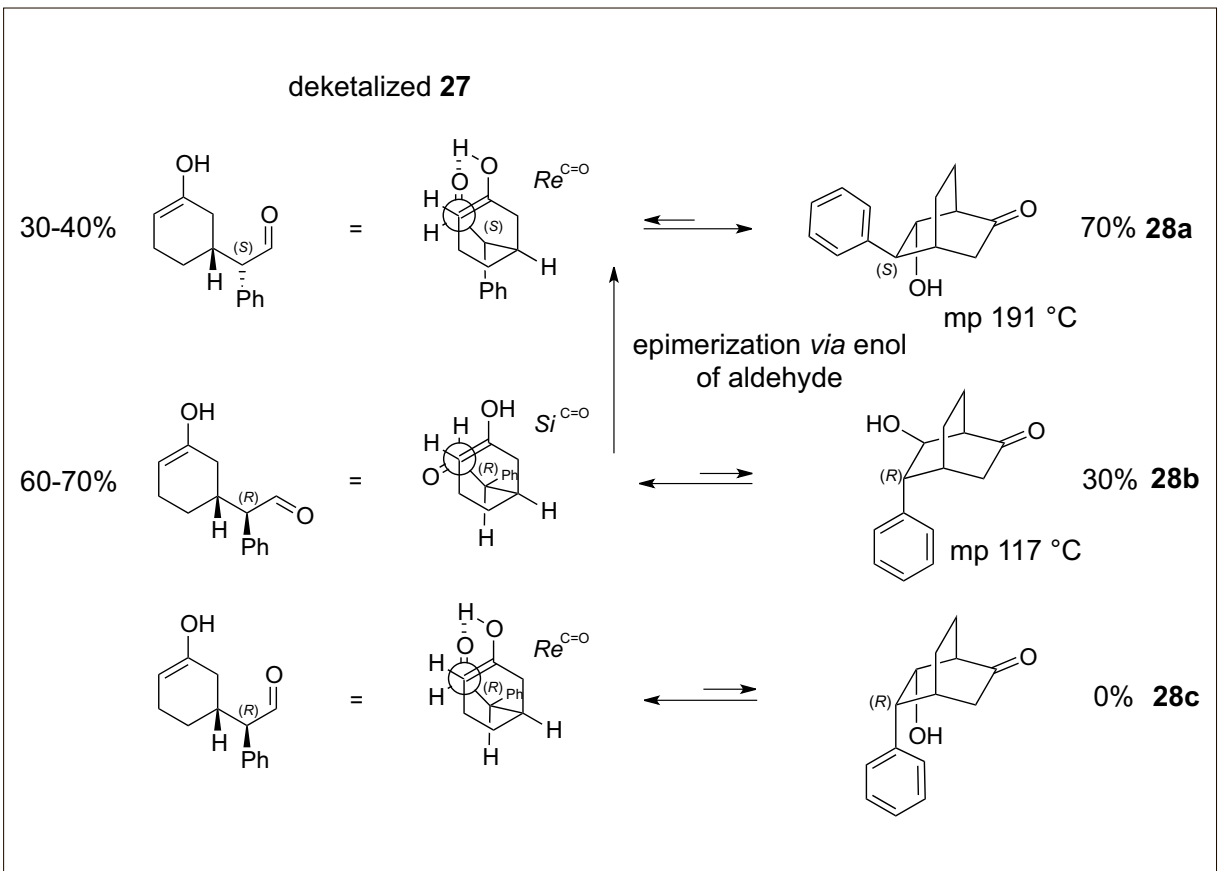

Scheme 11. CIDT in the intramolecular aldol reaction of deprotected 27 to secondary alcohols 28. The dr of the substrate $\mathbf{2 7}$ stems from the ester $\alpha$-arylation in the steps from $\mathbf{2 5}$ to 26 (see Scheme 9). 
excess (44\%) was acceptable as the upgrade to the desired er of 98:2 (for downstream chemistry towards ACT-280778) or $>99.5: 0.5$ was achieved by crystallization (Scheme 12). ${ }^{[8]}$ In contrast to the report by Bella et al., high selectivities were obtained without a cinchona alkaloid on top of chiral secondary amine organocatalysts, even in the absence of a base like Hünig's base.

Fig. 7 shows the pictures of a smallscale lab reaction (to 28a) to demonstrate the ease of processing. After achieving full conversion, the suspension was filtered at $20{ }^{\circ} \mathrm{C}$, and the filter cake was consecutively washed with water $(3 \times 0.5 \mathrm{~L})$ and toluene $(3 \times 0.4 \mathrm{~L})$. Drying under air for 2 h gave highly pure $\mathbf{1}$.

Potential transition states for the Michael addition to cyclohexenone are shown in Scheme 13 based on the seminal work published by List and Houk, [19] and Eschenmoser and Seebach, ${ }^{[20]}$ respectively. The distribution of the diastereoisomers 28a and 28b does not seem to be determined only by the steric and electronic factors governing the organocatalytic Michael-aldol reaction but also by the thermodynamic stability of the products based on the above equilibrium between 28b and 28a by retro-aldol reaction via epimerization of the benzylic position. The predominance of isomer 28a over $\mathbf{2 8 b}$ could hence result from the differences in solubility as discussed above (CIDT).

Table 1 compares the key attributes of three approaches to $\mathbf{1}$ that successfully served different purposes for the growing demands in the drug development process. Whereas the yield is similar for all the three routes with the number of steps ranging from 3 to 9 , the number of solvents went down to 3 and special technologies like running the scale-limited hazardous Diels-Alder reactions, handling of cyanides, or large-scale HPLC or Simulated Moving Bed (SMB) technology are no longer required. The major cost driver of the Diels-Alder route, i.e. the racemate resolution was successfully removed by the two $2^{\text {nd }}$ generation routes. The organocatalysis route runs without any metal that would call for expensive waste treatment on scale.

The overall reduction of the Cost of Goods (COG) for the manufacture of phenyl ketone 1 with the $2^{\text {nd }}$ generation route 2 (organocatalysis) as calculated on a 1 metric ton scale was impressive (>95\%) as compared to the Diels-Alder route (Fig. 8). The new process is operationally very simple and uses raw materials that are readily available and cheap. On industrial scale, the low cost of L-proline and the ease of upgrading the ee by crystallization more than compensates for the low enantiomeric excess. Designer organocatalysts that are often used at similar high loadings can

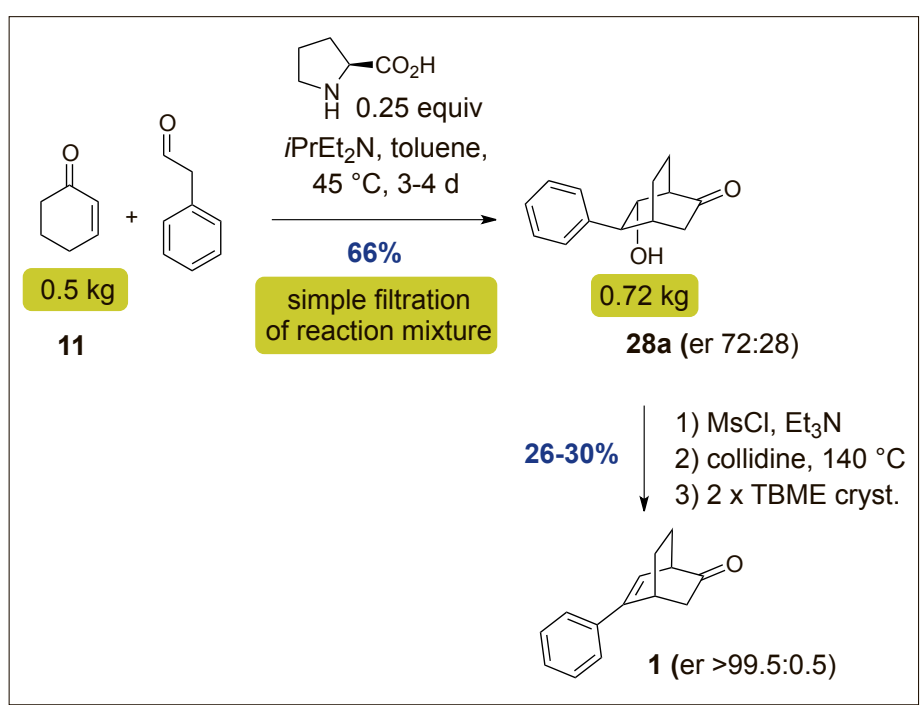

Scheme 12. Short and simple synthesis of bicyclic ketone 1 via organocatalysis.

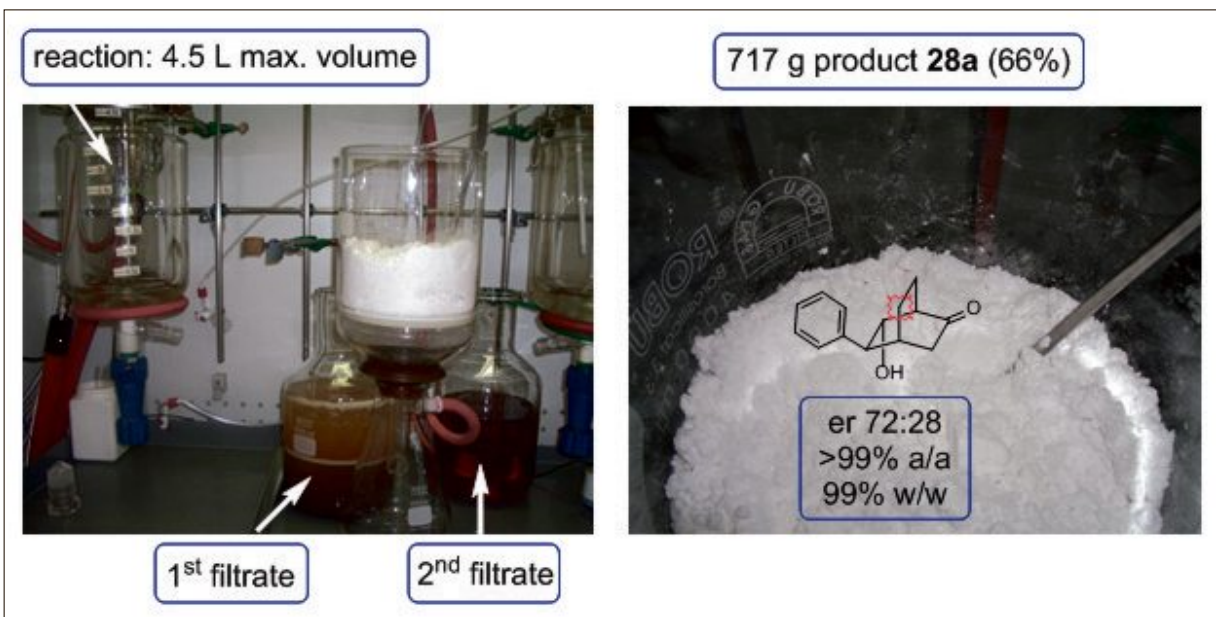

Fig. 7. Photographs of a $5-\mathrm{L}$ scale reaction using $0.5 \mathrm{~kg}$ cyclohexenone, $743 \mathrm{~g}$ phenylacetaldehyde, $45 \mathrm{~g}$ L-proline, $163 \mathrm{~g}$ Hünig's base in $3 \mathrm{~L}$ toluene. The two filtrates and the product $28 \mathrm{a}$ on the filter are displayed.

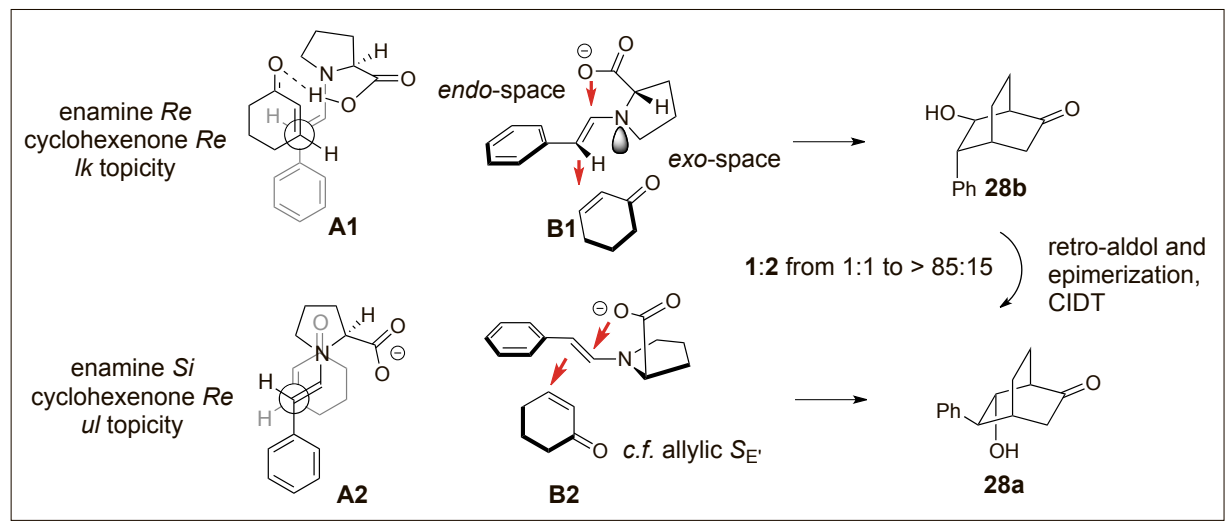

Scheme 13. Mechanistic models for the organocatalytic tandem Michael-aldol reaction. A1, A2 (List-Houk), B1, B2 (Seebach-Eschenmoser).

cause high production costs. It is noteworthy that the Shibasaki route led to $>60 \%$ reduction in $\mathrm{COG}$ even though the number of chemical steps increased from 3 to 9 . This apparent discrepancy stems from the low number of isolated intermediates (3), the high yields and selectivities combined with the ease of isolating and purifying the secondary bicyclic alcohol 28a. ${ }^{[7]}$

\section{Chiral Dienes}

Chiral dienes have gained considerable interest in metal-catalyzed asymmetric processes since 2003, notably for the catalytic enantioselective addition of arylboronates to unsaturated ketones or esters where these stable chiral ligands replace notably the chiral phosphines.[21] Besides 
Table 1. Route comparison to produce 1

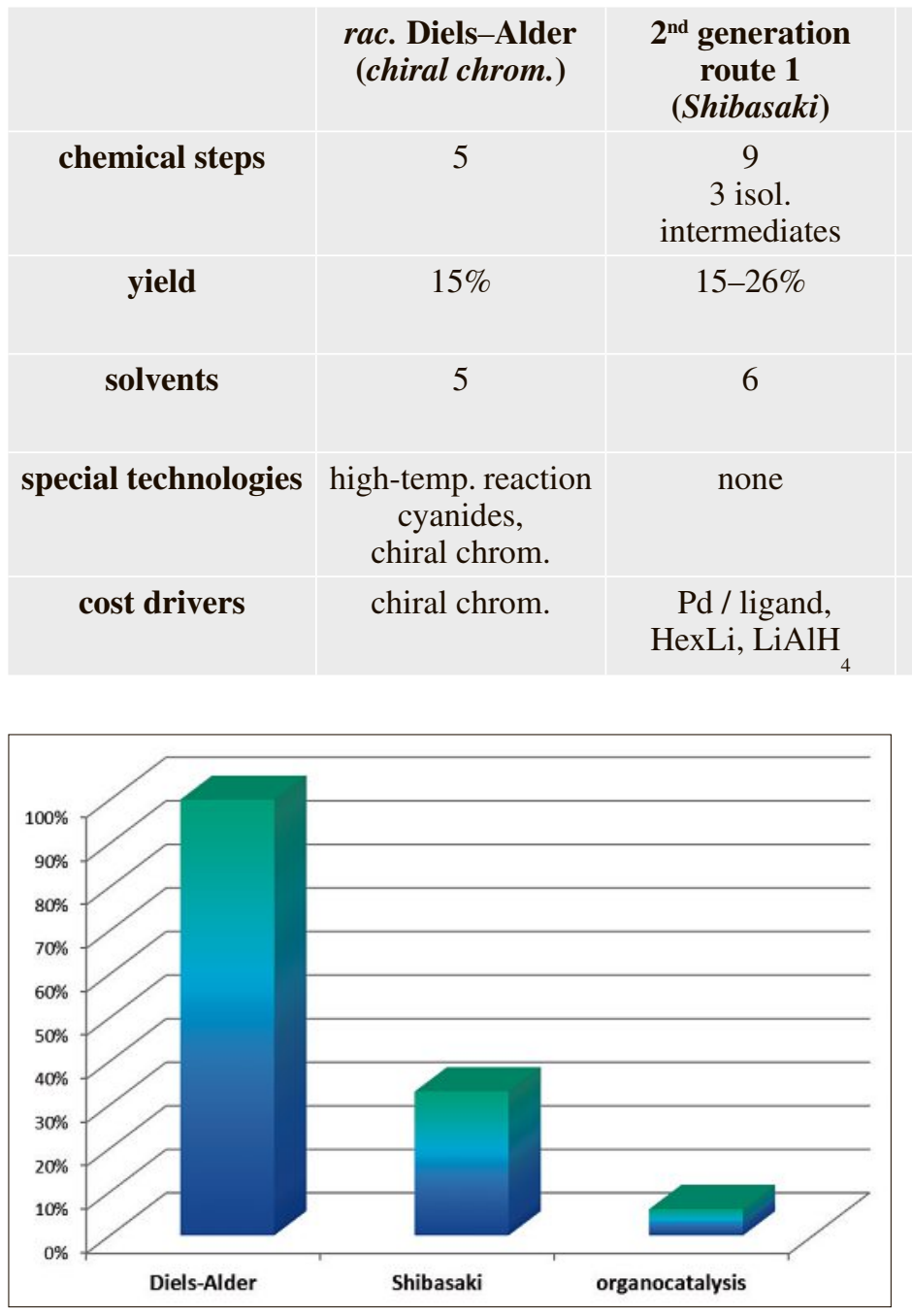

creased by a factor of approx. 10, whereas the PMI regarding the reagents decreased by a factor of $38 .{ }^{[23]}$ The operational simplicity, the low number of isolated intermediates, and the low cost of the organocatalyst (L-Pro) more than counterbalanced the low ee $(44 \%)$ of the organocatalytic step.

This set of ten $\mathrm{C} 1$-symmetric chiral bicyclo[2.2.2] octa-2,5-dienes has been tested as ligands in four $\mathrm{Rh}$-catalyzed arylation reactions: 1,4-addition to cyclopentenone, cyclohexenone, and tert-butyl cinnamate, and the 1,2-addition to $\mathrm{N}$-[(4nitrophenyl)-sulfonyl]imines. ${ }^{[24]}$ Scheme 16 depicts one of these reactions, the challenging 1,4-addition of arylboronic acids to tert-butyl cinnamate $\mathbf{3 1}$ that smoothly afforded the diphenylmethine $\mathbf{3 2}$ in high ee. Several of the novel dienes (30) gave higher enantioselectivities than the benchmark ligands reported in the literature.

Fig. 8. COG estimate on $1^{\prime} 000-\mathrm{kg}$ scale, ex-factory for 1 . Cost reduction of $>95 \%$ achieved. COG normalized at $100 \%$ for Diels-Alder approach.

\section{Conclusion}

This work exemplifies a recent paradigm in the pharmaceutical industry: companies, especially smaller or mid-sized ones, are no longer covering all major fields of expertise with regard to both the equipment and staff. Instead, they choose to tap into the vast pool of service providers available worldwide in order to focus their own resources on the strategically most pivotal assets. The screening capabilities and the excellent track record of Solvias AG helped us to find the best catalyst that other factors like stability and scope, the widespread use of any new class of ligands is influenced by their availability that is ultimately related to the ease and cost of their preparation. Specifically, Hayashi's bicyclo[2.2.2] octadiene (bod*) ligands 30a and 30b, which display a broad scope and excellent selectivities, are still lacking a simple synthesis in terms of scalability and costs (Scheme 14). ${ }^{[22]}$

Having secured access to large amounts of the ketone $\mathbf{1}$, we were intrigued to offer an easy and affordable access to this class of ligands as just two steps were required to synthesize Hayashi's bod* ligands from 1 (Scheme 15). ${ }^{[8]}$

Ten new C1-symmetric chiral dienes (30) were prepared (Fig. 9). This new and authoritative class of ligands is now accessible on kg-scale at much reduced costs. The feedback from academia was very positive, and we expect that these ligands will be used more frequently to fine-tune asymmetric catalysis.

The Process Mass Intensity (PMI: kg reagents required to produce $1 \mathrm{~kg}$ of final product), a metric commonly used to assess the efficiency of a process, was calculated for this new approach to Ph-bod*

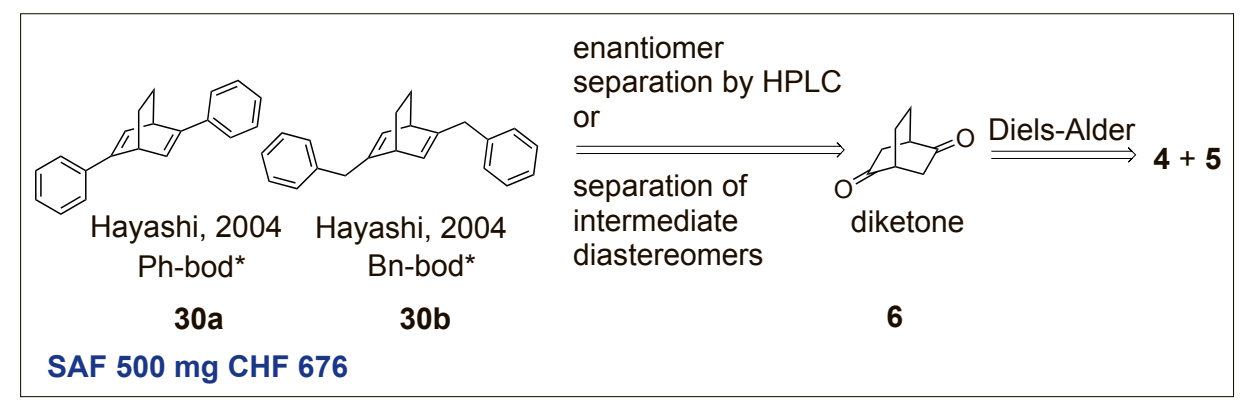

Scheme 14. Hayashi's bod* ligands and published syntheses thereof.

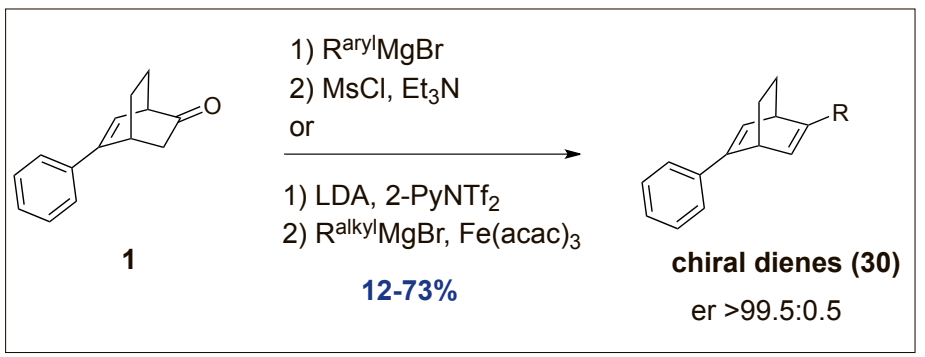

Scheme 15. Synthesis of novel chiral dienes (30) from now readily available chiral bicyclic ketone 1.

ligand 30a, starting from cyclohexenone. The PMI was calculated without solvents and water to account for the early research stage of the published route that was used for comparison. ${ }^{[22]}$ The overall yield has in- not only fulfilled acceptable performance but was of low cost. The use of the highly reactive Diels-Alder reagents and the reactions would not have been reduced to practice without the thorough safety as- 


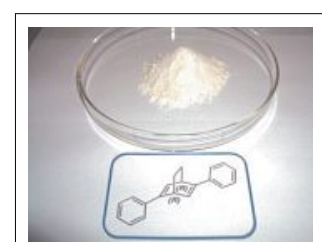

$(\mathrm{R}, \mathrm{R})-\mathrm{Ph}-$ bod $(30 \mathrm{a})$ 75-77 ${ }^{\circ} \mathrm{C}$ (Aldrich: $74-76^{\circ} \mathrm{C}$ )
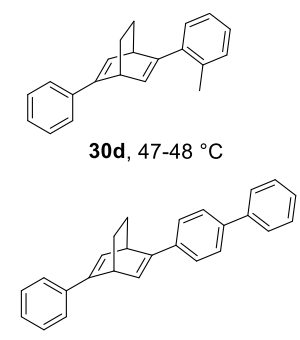

$30 \mathrm{~g}, 142^{\circ} \mathrm{C}(\mathrm{dec}$.)
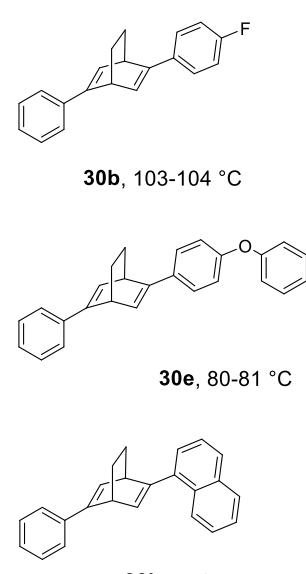

30h, resin
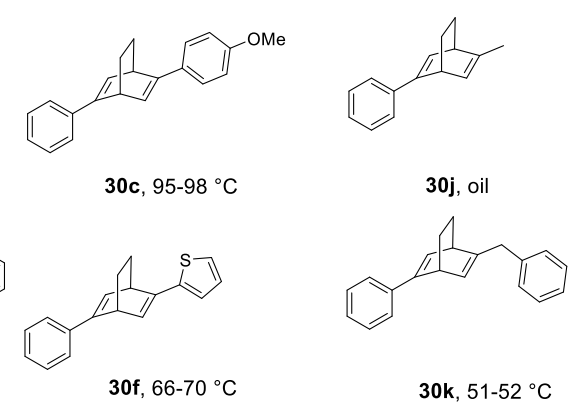

30c, $95-98^{\circ} \mathrm{C}$

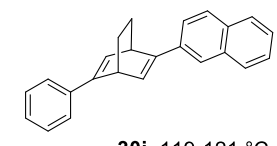

30i, $119-121^{\circ} \mathrm{C}$
Fig. 9. Melting points of novel dienes (30); er $>99.5: 0.5$, purity $>99 \%$ a/a (HPLC).

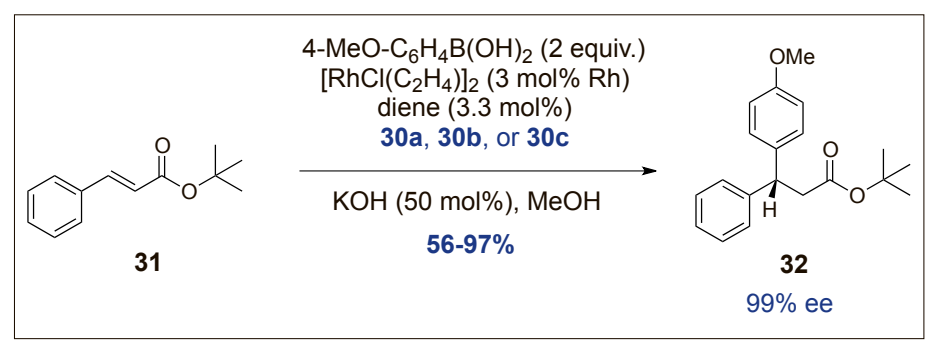

Scheme 16. One of the four Rh-catalyzed arylation reactions used for benchmarking of the new C1-symmetric diene ligands $30 \mathrm{~b}$ and $30 \mathrm{c}$ with 30a (Ph-bod*).

sessments with the Swissi Process Safety $\mathrm{GmbH}$. Both activities ran in parallel to our own development, and response time was key. Certainly, the vicinity of both partners to Actelion was of help. Another trend that is showcased in the present work is the increasing collaboration of industry and academia. Gone are the times where chemical and pharmaceutical companies heavily invested into their own fundamental research ('Zentralforschungslaboratorien', at Ciba, Merck, or BASF) that was turned into magnificent large-scale technical applications. Explorative research is abundant in academia, and we think that industrial process research groups need to consider it with an unbiased attitude. The realization of a flow application for the high temperature Diels-Alder reaction with Dr. Roger Marti is an excellent example for such a successful team work. The result proved to be a technical contingency solution if the batch mode had not been successfully scaled up. A collaboration with the Ecole d'Ingénieurs et d'Architectes de Fribourg led to the application of a dozen of new chiral dienes in catalytic asymmetric reactions.

Balancing the short-term benefit of a fit-for-purpose route with the long-term benefit of a route destined for ton-scale manufacturing is the process chemist's constant challenge. On the long run, the goal is to reduce the time from selection of the clinical candidate to Entry-into-
Man (EIM), the Phase 1 studies, and, ultimately, to market. If a robust route can be established early in drug development, this will save later expenses arising from trouble shooting on the manufacturing floor. Late route changes are associated with a higher regulatory burden. Another argument to invest into early Process R\&D is that route development close to the first $\mathrm{kg}$-batches is typically more expeditious due to the immediate availability of both the process know-how and ideas of the involved chemists and the intermediates at the site of manufacture. This frontloading investment also fulfills the $\mathrm{QbD}$ requirements purported by the FDA: the earlier a robust route is designed and developed, the shorter is the overall time and investment until the process understanding meets the stringent requirements for validation and registration.

\section{Acknowledgments}

This work was only made possible by the professional help of a multitude of highly talented coworkers. We are deeply indebted to their enthusiasm and dedication. Roman Inauen deserves a special thank for his key role in carrying out the lab experiments for the $2^{\text {nd }}$ generation processes. We thank Arran, (now Almac) Valsynthese, Chiral Technologies, Novasep, and Alcami (formerly known as CML) for the support during production. We are indebted to Dr. Kurt Hilpert, Dr. Dorte Renneberg, and Dr. Thomas Weller from Medicinal Chemistry for the close-knit collaboration. S.A. warmly thanks Prof. Dieter Seebach for fruitful discussions.

Received: May 13, 2016

[1] K. Hilpert, F. Hubler, D. Renneberg, PCT Int Appl. WO/2008/132679, 2008.

[2] R. K. Hill, G. H. Morton, J. R. Peterson, J. A. Walsh, L. A. Paquette, J. Org. Chem. 1985, 50, 5528 .

[3] K. Ikai, K. Takeuchi, T. Kinoshita, K. Haga, K. Komatsu, K. Okamoto, J. Org. Chem. 1991, 56, 1052.

[4] Y. Otomaru, K. Okamato, R. Shintani, T Hayashi, J. Org. Chem. 2005, 70, 2503.

[5] J.-A. Funel, G. Schmidt, S. Abele, Org. Process Res. Dev. 2011, 15, 1420.

[6] Y. Xu, K. Ohori, T. Ohshima, M. Shibasaki, Tetrahedron 2002, 58, 2585.

[7] S. Abele, R. Inauen, J.-A. Funel, T. Weller, Org. Process Res. Dev. 2012, 16, 129.

[8] S. Abele, R. Inauen, D. Spielvogel, C. Moessner, J. Org. Chem. 2012, 77, 4765.

[9] S. Abele, M. Schwaninger, H. Fierz, G. Schmidt, J.-A. Funel, F. Stoessel, Org. Process Res. Dev. 2012, 16, 2015.

[10] S. Abele, G. Schmidt, J.-A. Funel, M. Schwaninger, S. Wagner, in 'Managing Hazardous Reactions and Compounds in Process Chemistry', Eds. J. A. Pesti, A. F. Abdel-Magid, ACS Symposium Series, American Chemical Society: Washington, DC, 2014, vol 1181, p. 189.

[11] S. Abele, S. Höck, G. Schmidt, J.-A. Funel, R. Marti, Org. Process Res. Dev. 2012, 16, 1114.

[12] Chiralpak AS-V, MeOH, 0.1\% Et N, $>90 \%$ recovery of both enantiomers, er $>99: 120 \times 25$ cm column, $5 \mathrm{~kg}$ CSP, $216 \mathrm{~L} / \mathrm{h}$, conc.: $200 \mathrm{~g} / \mathrm{L}$, 41 g per injection. $>2.7 \mathrm{~kg} 1 / \mathrm{kg}_{\mathrm{CSP}} / \mathrm{d}$ (CSP: chiral stationary phase).

[13] a) J.-A. Funel, S. Abele, Angew. Chem. Int. Ed. 2013, 52, 3822; b) J.-A. Funel, S. Abele, Angew. Chem. 2013, 125, 3912.

[14] J.-A. Funel, S. Brodbeck, Y. Guggisberg, R. Litjens, T. Seidel, M. Struijk, S. Abele, Org. Process Res. Dev. 2014, 18, 1674.

[15] DSC of a mixture of both regioisomers 20 shows an exotherm with left limit $290^{\circ} \mathrm{C}$, peak $338^{\circ} \mathrm{C}$, and the decomposition energy of -3154 $\mathrm{kJ} / \mathrm{kg}$.

[16] a) S. Abele, J.-A. Funel, WO 2012/052943 (publication 26.04.2012); b) S. Abele, J.A. Funel WO 2012/052939 (publication 26.04.2012).

[17] CIDT is a special type of dynamic kinetic resolution with recycling of the undesired isomer, see: K. M. J. Brands, A. J. Davies, J. Chem. Rev. 2006, 106, 2711.

[18] M. Bella, D. M. Scarpino Schietroma, P. P. Cusella, T. Gasperi, Chem. Commun. 2009, 597.

[19] S. Mukherjee, J. W. Yang, S. Hoffmann, B. List, Chem. Rev. 2007, 107, 5471.

[20] D. Seebach, A. K. Beck, D. M. Badine, M. Limbach, A. Eschenmoser, A. M. Treasurywala, R. Hobi, Helv. Chim. Acta 2007, 90, 425.

[21] a) T. Hayashi, K. Ueyama, N. Tokunaga, K. Yoshida, J. Am. Chem. Soc. 2003, 125, 11508; b) R. Shintani, T. Hayashi, Aldrichimica Acta 2009, 42, 31; c) C. Defieber, H. Grützmacher, E. M. Carreira, Angew. Chem. Int. Ed. 2008, 47, 4482.

[22] Y. Otomaru, K. Okamato, R. Shintani, T. Hayashi, J. Org. Chem. 2005, 70, 2503.

[23] PMI without solvents and water: 1365 for published route, and 36 for novel organocatalytic approach; solvent usage (10174 vs. 207), yield (1 vs. $11 \%$ ).

[24] R. Brönnimann, S. Chun, R. Marti, S. Abele, Helv. Chim. Acta 2012, 95, 1809. 